\title{
Is the time constant of the pulmonary circulation truly constant?
}

\author{
To the Editor:
}

I read with great interest the recent article by DELCROIX et al. [1] on vascular and right ventricular remodelling in chronic thromboembolic pulmonary hypertension (CTEPH). I found it very informative; however, I would like to comment on some of the statements that were made. In relation to pulmonary vascular remodelling, the authors have stated that in spite of there being proportionally more wave reflection in $\mathrm{CTEPH}$, the $\mathrm{RC}$ (resistance $\times$ compliance) time constant of the pulmonary circulation is not different in CTEPH and pulmonary arterial hypertension. They invoked that the functional adaptation of the pulmonary circulation to vascular disease is monotonous (any change in pulmonary vascular resistance (PVR) determines proportional changes in pulmonary compliance $(C \mathrm{p}))$. Therefore, compliance and resistance are inseparably connected in health and disease, and after treatment [2]. The constant RC time also explains the proportional relationships between systolic and diastolic pulmonary arterial pressure (PAP) with mean pressure being linear relationships with a slope (Ksys and Kdias, respectively) and intercept $[3,4]$. Moreover, a constant $\mathrm{PVR} \times C_{\mathrm{p}}$ product implies that right ventricular oscillatory power ( $W_{\mathrm{osc}}$ ) remains a constant fraction of total power $\left(W_{\text {tot }}\right)$, irrespective of PAP [3]. As mean PAP (mPAP) is the geometric mean of systolic PAP (sPAP) and diastolic PAP (dPAP), the product of Ksys and Kdias is close to unity, so that $\mathrm{mPAP}=\sqrt{ }(\mathrm{sPAP} \times \mathrm{dPAP})$. This relationship, however, is independent of a constant RC time [4].

TEDFORD et al. [5] have recently challenged the concept of a constant RC time. They showed that an increased pulmonary capillary wedge pressure (PCWP) in patients with passive pulmonary hypertension and left heart failure appears to proportionally shorten the RC time constant (PCWP $\leqslant 10 \mathrm{mmHg}$, RC time $0.43 \pm 0.15 \mathrm{~s} ;$ PCWP $\geqslant 20 \mathrm{mmHg}$, RC time $0.28 \pm 0.12 \mathrm{~s}$ ) and relatively increase in right ventricular afterload, contributing to right ventricular dysfunction.

CTEPH has been recognised as a "dual" pulmonary vascular disorder, consisting of a large-vessel vascular remodelling process of thrombus organisation combined with a small-vessel vascular disease. Isobaric steady-component analysis allowed us to differentiate the pulsatile component between idiopathic pulmonary arterial hypertension (IPAH) and CTEPH [6]. Pre-operative operable CTEPH is characterised by a more predominant wave reflection than IPAH. This explains the disproportionately lower $C_{\mathrm{p}}$ with a downward and leftward displacement of the inverse hyperbolic relationship between PVR and $C_{p}$ in the CTEPH patients, and a significantly lower RC time with respect to the IPAH cohort $(0.5 \pm 0.13$ versus $0.6 \pm 0.3 \mathrm{~s}$ ) [6]. Accordingly, operable CTEPH increased mPAP with an increase in sPAP that was proportionally more than in IPAH patients, while dPAP increased less (fig. 1a) [7]. Therefore, dynamic right ventricular afterload in CTEPH patients was higher than IPAH and correlated with the higher right ventricular end-diastolic diameter [6]. We also confirmed that mPAP is the geometric mean of sPAP and dPAP, as in IPAH (fig. 1b). Accordingly, MACKENZIE Ross et al. [8] have recently analysed the relationship between PVR and $C \mathrm{p}$ in patients with IPAH and proximal CTEPH. They showed that, in preendarterectomy CTEPH, $C_{\mathrm{p}}$ decreases proportionally more than PVR increases because of increased wave reflection secondary to proximal obstructions. Therefore, they concluded that the proximal CTEPH patients present a pulmonary RC time constant decrease with respect to IPAH. This is in accord with a wider pulse pressure and greater dynamic right ventricular afterload.

RV $W_{\text {tot }}$ is the sum of steady power $\left(W_{\mathrm{s}}\right)$ and $W_{\mathrm{osc}}\left(W_{\mathrm{tot}}=W_{\mathrm{s}}+W_{\mathrm{osc}}\right)$. $W_{\mathrm{s}}$ corresponds to the energy required to move blood forward and maintain cardiac output. Wosc represents the extra energy wasted to accelerate blood in a pulsatile fashion and is related directly to the amount of wave reflection. Wosc can be expressed as a fraction of $W_{\text {tot }}\left(W_{\text {osc }} / W_{\text {tot }}\right.$ ). The higher the $W_{\text {osc }} / W_{\text {tot }}$, the greater the waste of $W_{\text {tot }}$ on pulsatile work. We demonstrated that during proximal pulmonary hypertension (main pulmonary artery ensnarement), $W_{\text {osc }} / W_{\text {tot }}$ and the energy transfer from the right ventricle to the pulmonary circulation decrease, reflecting an impairment of the ventricular-vascular coupling [9]. In agreement with us, PAgnAmENTA et al. [10] showed that both the pulsatile component of hydraulic work and $W_{\text {osc }} / W_{\text {tot }}$ increased after pulmonary artery ensnarement and this is associated with a markedly shorter RC time.

Despite the apparent contradiction between the article by Delcroix et al. [1] and that by MacKenzie Ross et al. [8], the CTEPH patients included in Lankhaar cohort were inoperable, with probably distal CTEPH 

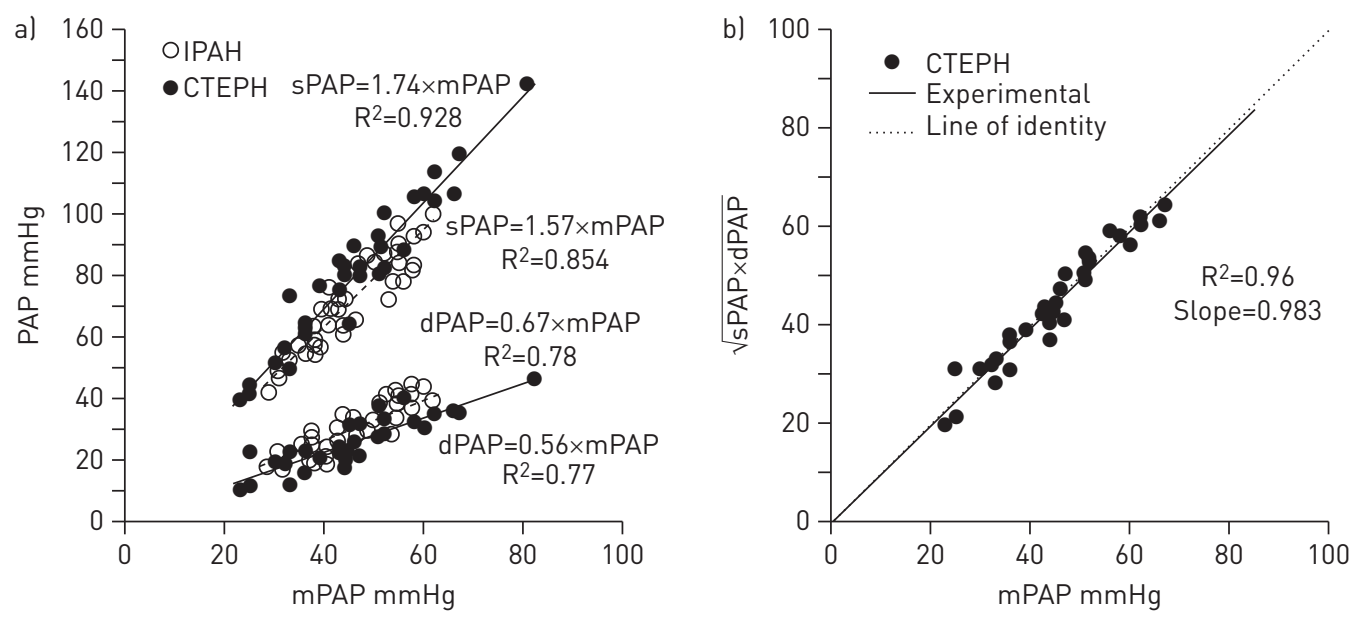

FIGURE 1 a) Correlation between systolic pulmonary arterial pressure (sPAP) and diastolic pulmonary arterial pressure (dPAP) and mean pulmonary arterial pressure (mPAP) in idiopathic pulmonary arterial hypertension (IPAH) and chronic thromboembolic pulmonary hypertension (CTEPH). b) Relationship between mPAP and $\sqrt{(s P A P} \times \mathrm{dPAP})$. All points are close to the line of identity.

and a predominant small vessel vasculopathy (very similar to IPAH). The dual pulmonary vascular disorder determines a wide spectrum of loading conditions in CTEPH. Therefore, the decreased $C_{\mathrm{p}}$ is not necessarily proportional to the increased PVR. A reduced RC time constant in operable CTEPH compared with IPAH equates to a faster pressure decay in diastole, changing the proportionality of sPAP and dPAP with mPAP (fig. 1a). This is associated with a lower pulmonary arterial cushioning performance in CTEPH patients and an impairment of the right ventricular-vascular coupling.

In conclusion, RC time of the pulmonary circulation is not always constant; it can become shorter in pulmonary hypertension: not only due to a stiffer pulmonary arterial tree caused by increased pulmonary venous pressure (pulmonary hypertension with left heart failure) but also an increased wave reflection caused by the presence of proximal pulmonary obstructions (operable CTEPH), shortened RC time, increased dynamic right ventricular afterload at the expense of the pulsatile component and impairment of ventricular-vascular coupling.

0

@ERSpublications

Time constant of the pulmonary circulation shortens in pulmonary hypertension due to increased pulsatile afterload http://ow.ly/qy9Ju

Juan C. Grignola

Dept of Pathophysiology, Hospital de Clínicas, School of Medicine, Universidad de la República, Montevideo, Uruguay.

Correspondence: J.C. Grignola, Dept of Pathophysiology, Hospital de Clínicas, School of Medicine, Universidad de la República, Avda Italia 2870, PC 11300 Montevideo, Uruguay. E-mail: jgrig@fmed.edu.uy

Received: Sept 082013 | Accepted: Oct 062013

Conflict of interest: None declared.

\section{References}

1 Delcroix M, Vonk-Noordegraaf A, Fadel E, et al. Vascular and right ventricular remodelling in chronic thromboembolic pulmonary hypertension. Eur Respir J 2013; 21: 1046-1049.

2 Lankhaar J, Westerhof N, Faes TJC, et al. Pulmonary vascular resistance and compliance stay inversely related during treatment of pulmonary hypertension. Eur Heart J 2008; 29: 1688-1695.

3 Saouti N, Westerhof N, Postmus PE, et al. Right ventricular oscillatory power is a constant fraction of total power irrespective of pulmonary artery pressure. Am J Respir Crit Care Med 2010; 182: 1315-1320.

4 Kind T, Faes TJC, Vonk-Noordegraaf A, et al. Proportional relations between systolic, diastolic and mean pulmonary artery pressure are explained by vascular properties. Cardiovasc Eng Technol 2011; 2: 15-23.

5 Tedford RY, Hassoun PM, Mathai SC, et al. Pulmonary capillary wedge pressure augments right ventricular pulsatile loading. Circulation 2012; 125: 289-297.

6 Grignola JC, Ruiz-Cano MJ, Escribano P, et al. Isobaric analysis of pulmonary arterial compliance of idiopathic and chronic thromboembolic pulmonary hypertension: correlation with right ventricular remodeling. Eur Heart J 2009; 30: 108.

7 Grignola JC, Domingo E. Pulmonary endarterectomy in chronic thromboembolic pulmonary hypertension: How can patients be better selected? World J Cardiol 2013; 26: 18-21. 
8 MacKenzie Ross RV, Toshner MR, Soon E, et al. Decreased time constant of the pulmonary circulation in chronic thromboembolic pulmonary hypertension. Am J Physiol 2013; 305: H259-H264.

9 Grignola JC, Bia D, Ginés F, et al. Improved right ventricular-vascular coupling during active pulmonary hypertension. Int J Cardiol 2007; 115: 171-182.

10 Pagnamenta A, Vanderpool R, Brimioulle S, et al. Proximal pulmonary arterial obstruction decreases the time constant of the pulmonary circulation and increases right ventricular afterload. J Appl Physiol 2013; 114: 1586-1592.

Eur Respir J 2014; 43: 1539-1541 | DOI: 10.1183/09031936.00158113 | Copyright @eERS 2014

From the authors:

J.C. Grignola points out that the product of pulmonary vascular resistance by pulmonary vascular compliance, or time constant (RC time) of the pulmonary circulation is not truly constant, and that this impacts on the pulsatile component ( $W_{\text {osc }}$ ) of right ventricular hydraulic load ( $W_{\text {tot }}$ ).

$\mathrm{He}$ is right. But the question is, by how much? In the experimental study by PAGNAmenta et al. [1], pulmonary hypertension induced by ensnarement of the pulmonary arteries, to mimic purely proximal chronic thromboembolic pulmonary hypertension (CTEPH), was associated with a decrease in RC time from $0.5 \mathrm{~s}$ to $0.3 \mathrm{~s}$, while microembolism to mimic purely distal obstruction, like in pulmonary arterial hypertension (PAH), was associated with an increase in RC time from $0.5 \mathrm{~s}$ to $0.8 \mathrm{~s}$. Associated changes in $W_{\text {osc }} / W_{\text {tot }}$ went from $25 \%$ to $29 \%$ and $16 \%$, respectively. These would be the extreme possible deviations of a fixed $W_{\text {osc }} / W_{\text {tot }}$ of $23 \%$ calculated for all types and severities of pulmonary hypertension by SAOUTI et al. [2] on the basis of recovered constant RC times. In other words, $W$ tot, as an estimation of right ventricular afterload, is not always equal to $1.3 \times$ steady-flow $W$ (calculated as the product of mean pulmonary artery pressure and stroke volume) proposed by SAOUTI et al. [2], but in the range 1.2-1.4. This is not much. Furthermore, as also underscored by J.C. Grignola [1], vascular obstruction in clinical pulmonary hypertension tends to be inhomogeneous and spread over the entire vascular tree, especially in CTEPH, so that the variations of the estimate of $W$ tot would be markedly smaller, probably reduced to somewhere between 1.25 and $1.35 \times$ steady-flow $W$. This corresponds to trivial variations in afterload. Unsurprisingly, as illustrated in figure 1a of J.C. Grignola's correspondence, the reported range of RC times has a minimal effect on the proportionality of systolic, mean and diastolic pulmonary artery pressures. Conversely, the near-constant RC time in pulmonary hypertension is still in marked contrast with the extensive variability of the systemic RC time [3].

The discovery of "laws of nature" in medical research clarifies understanding and is essential for bedside translation of bench research. The repeated observations by LANKHAAR et al. [4] that RC time is constant in the pulmonary circulation has helped considerably in the assessment of right ventricular afterload in pulmonary hypertension. But scientific truth has to be constantly rechallenged, and uncovering exceptions leads to developments of new paradigms and innovation. J.C. Grignola is to be commended for pointing out that the RC time is shortened in left heart failure with increased pulmonary venous pressure, which makes the pulmonary circulation less compliant at any given level of resistance [3], and in operable CTEPH because of increased wave reflection and proximal arterial stiffness [5]. However, the deviations are small, so that the general rule of the constancy of the RC time remains a reasonable approximation in all circumstances.

Thus, while we agree with J.C. Grignola's pertinent remarks, we humbly feel allowed to maintain our statement that the RC time of the pulmonary circulation is approximately the same in CTEPH and PAH [6], indicating surprisingly little impact of proximal obstruction-related increased wave reflection or stiffness on right ventricular afterload.

0

@ERSpublications

RC time of the pulmonary circulation is approximately the same in CTEPH and PAH http://ow.ly/tVvcs

Robert Naeije $^{1}$ and Marion Delcroix ${ }^{2}$

${ }^{1}$ Dept of Cardiology, Erasme University Hospital, Brussels, and ${ }^{2}$ Dept of Pneumology, University Hospitals of Leuven, Leuven, Belgium.

Correspondence: R. Naeije, Faculty of Medicine, Free University of Brussels, 808 Route de Lennik, Brussels 1070, Belgium. E-mail: rnaeije@ulb.ac.be

Received: Oct 062013 | Accepted: Oct 082013

Conflict of interest: Disclosures can be found alongside the online version of this article at www.erj.ersjournals.com 